Objective The dissemination and active support for clinical practice guidelines development method based on the principle of EBM, is one of the main pillars of MINDS (Medical Information Network Distribution Service) consignment project for EBM promotion implemented by Ministry of Health, Labour and Welfare Japan. In 2007, with the publication of MINDS guidance to develop Clinical practice guidelines 2007 by Fukui et al., a protocol to develop clinical practice guidelines was introduced as the most valid manual at that time in Japan. However, advanced improvements towards the global standards in the CPG development methodology have been established within these 5 years after previous publication, such as GRADE system, AGREE II, IOM etc. Accordingly, the Workshop for Clinical Practice Guidelines development methodology is planned to introduce the latest advanced methodology for medical and healthcare professionals who engaged in the clinical practice guidelines development. 2. Contents This workshop comprises the lectures and group practices, assuming AGREE II as a standard for the educational package development and, using GRADE system for quality of evidence and strength of recommendation, which promote the CPG development methods for disease management. We hope to show the 'PROGRAMME' for the progress of the workshop at G-I-N2013. 3. Target Audience For all medical and healthcare professionals who have interest in and for those who engage in clinical practice guidelines development in Japan. 4. Workshop Outline Educational workshops will be held four times within a year 2013 in Tokyo Japan. The first workshop date: 2013-03-20 (Wed) 10:00 17:00

\section{P326 IMPLEMENTING AN AUDIT AND FEEDBACK INTERVENTION TO REDUCE ANTIBIOTIC PRESCRIBING IN GENERAL DENTAL PRACTICE}

${ }^{1} \mathrm{~L}$ Young, ${ }^{1} \mathrm{D}$ Stirling, ${ }^{2} \mathrm{P}$ Elouafkaoui, ${ }^{1} \mathrm{~S}$ Rutherford, ${ }^{3} \mathrm{C}$ Ramsay, ${ }^{2,1} \mathrm{~J}$ Clarkson. ${ }^{1} T$ RiaDS, Research Methodology Group, NHS Education for Scotland, Dundee, UK; ${ }^{2}$ University of Dundee, Dundee, UK; ${ }^{3}$ University of Aberdeen, Aberdeen, UK

\section{0:1136/bmjgs-2013-002293.257}

Background In Scotland, guidance for prescribing in dentistry was published in 2008 in response to concerns about over-prescribing of antibiotics. The guidance recommends that antibiotic prescribing must be kept to a minimum. However, evidence from routinely collected data demonstrates that dental antibiotic prescribing is steadily increasing, now accounting for $9 \%$ of primary care antibiotic prescriptions.

Objectives To compare the effectiveness of different audit and feedback $(\mathrm{A} \& \mathrm{~F})$ strategies for the implementation of recommendations on dental antibiotic prescribing.

Methods The study is an 18 month, three-arm randomised controlled trial. All dentists in Scotland are being randomised to either: 1) access to a pre-approved national audit; 2) access to the audit plus individualised feedback; 3) access to the audit, individualised feedback and a persuasive message. The primary outcome is the number of antibiotic prescriptions per 100 patients. A process evaluation will be conducted to elucidate the mechanisms by which $A \& \mathrm{~F}$ influences practice. The trial was developed in collaboration with system-level stakeholders from policy, education and service providers.

Results Development of all interventions is complete and the trial will begin in March 2013. Processes are being developed to enable integration of the most effective intervention into national systems intended to reduce antibiotic prescribing.
Discussion Collaboration with system-level stakeholders has helped ensure that the trial addresses national priorities and has engendered system-level action for national implementation of the most effective intervention.

Implications for Guideline Developers/Users Generating robust experimental evidence on an intervention's effectiveness and collaboration with system-level stakeholders can increase the likelihood of its adoption as a policy initiative.

\section{P327 OVERVIEW OF CLINICAL PRACTICE GUIDELINES IN JAPAN - FROM THE POINT OF VIEW OF PATIENT- INVOLVEMENT}

H Suzuki. The International Medical Information Center, Tokyo, Japan

\section{0:1136/bmjqs-2013-002293.258}

Background In Japan, the official movement to develop Clinical Practice Guidelines (CPGs) began in 1999 with the financial support of the Ministry of Health, Labour and Welfare -MHLW). Since then, CPGs in various fields have been developed and development methods using the principles of evidence-based medicine are becoming popular.

Objectives The First objective of the study is to clarify how many CPGs developed and published in Japan in 2013. The second is to make clear if patient-involvement is popular or not.

Methods I have searched the existing CPGs comprehensively. Out of the 600 searched CPGs, well-formulated ones were selected if they met the following criteria: defining clinical questions to be addressed, reviewing evidence, determinig grade of recommendation, and becoming open to the public. I have checked public involvement in developing.

Results Nevertheless 120 CPGs are selected, the number of CPGs, containing patient/carer member in developing process, is only 6 . And the number of patient-version CPGs is only 29 .

Discussion A reason why the patient-involvement doesn't become popular, came from MHLW decision, stopping the financial support in 2004. This decision resulted in the view that individual professional societies were required to develop CPGs applicable to those topics related to their societies. Accordingly, a given professional society, which should always be involved in the CPG development group as one of the main stakeholders, was the sole party involved in the development of CPGs.

\section{P329 DEVELOPING A STRATEGY TO ASSESS THE REPORTING OF THE UPDATING PROCESS IN CLINICAL PRACTICE GUIDELINE: A DRAFT CHECKLIST}

1,2R Vernooij, 1,2A Sanabria, 1,2 L Martínez García, ${ }^{3} \mathrm{~J}$ Makarski, ${ }^{3} \mathrm{M}$ Brouwers, ${ }^{1,2} \mathrm{P}$ AlonsoCoello. 'Iberoamerican Cochrane Centre, Barcelona, Spain; ${ }^{2}$ Institute of Biomedical Research (IIB Sant Pau), Barcelona, Spain; ${ }^{3}$ Department of Oncology, McMaster University, Hamilton, Canada

\section{0:1136/bmjqs-2013-002293.259}

Background Scientific knowledge is in constant change and, therefore, clinical practice guidelines (CPGs) require a frequent reassessment. However, the best methodology for updating CPGs is not known and methods are poorly reported in CPGs. A framework to evaluate the quality of reporting the updating process in CPGs and to provide guidance for minimum thresholds for an updating strategy is needed.

Objective To develop a CPG update reporting checklist. 